\title{
THE EXTENDED MAPPING CLASS GROUP CAN BE GENERATED BY TWO TORSIONS
}

\author{
XIAOMING DU
}

\begin{abstract}
Let $S_{g}$ be the closed oriented surface of genus $g$ and let $\operatorname{Mod}^{ \pm}\left(S_{g}\right)$ be the extended mapping class group of $S_{g}$. When the genus is at least 5 , we prove that $\operatorname{Mod}^{ \pm}\left(S_{g}\right)$ can be generated by two torsion elements. One of these generators is of order 2 , and the other one is of order $4 g+2$.
\end{abstract}

\section{INTRODUCTION}

Let $S_{g}$ be the closed oriented surface of genus $g$. The extended mapping class group $\operatorname{Mod}^{ \pm}\left(S_{g}\right)$ is defined as $\operatorname{Homeo}^{ \pm}\left(S_{g}\right) / \operatorname{Homeo}_{0}\left(S_{g}\right)$, the group of homotopy classes of homeomorphisms (including orientation-preserving ones and orientation-reversing ones) of $S_{g}$, and the mapping class group $\operatorname{Mod}\left(S_{g}\right)$ is defined by Homeo ${ }^{+}\left(S_{g}\right) / \mathrm{Homeo}_{0}\left(S_{g}\right)$, the group of orientation-preserving homotopy classes of homeomorphisms of $S_{g}$.

For $\operatorname{Mod}\left(S_{g}\right)$, Dehn and Lickorish found independently Dehn twist generating sets of $\operatorname{Mod}\left(S_{g}\right)$ [3, 11]. Humphries reduced the number of Dehn twist generators to the lowest bound [5]. Wajnryb in [17] found that the minimal number of the generators (not only Dehn twist generators) for the mapping class groups is 2 .

We are also interested in the torsion generating set. McCarthy and Papadopoulos in [15] proved that $\operatorname{Mod}\left(S_{g}\right)$ can be generated by infinitely many elements of order 2 when $g \geq 3$. Luo showed that $\operatorname{Mod}\left(S_{g}\right)$ is generated by $12 g+6$ elements of order 2 when $g \geq 3[12]$. Brendle and Farb reduced the number of the involution generators to 6 [1] and prove $\operatorname{Mod}\left(S_{g}\right)$ can be generated by three torsion elements. Kassabov reduced the number of the involution generators to 4 [8] for $g \geq 7$. Korkmaz in [9] proved $\operatorname{Mod}\left(S_{g}\right)$ can be generated by two torsion elements of order $4 g+2$. Monden in [14] proved $\operatorname{Mod}\left(S_{g}\right)$ can be generated by 3 torsion elements of order 3 .

2010 Mathematics Subject Classification. 57N05, 57M20, 20F38.

Key words and phrases. mapping class group, generator, torsion.

This research is supported by NSFC (Grant No. 11401219). The author wish to thank BICMR for its hospitality and thank Boju Jiang who asked me about the problem of finding lower bounds of the orders and the numbers of torsion generators for the mapping class groups. The author also wish to thank Dingsen Yan who teach me how to draw pictures with the metapost package of the latex. 
For the extended mapping class group $\operatorname{Mod}^{ \pm}\left(S_{g}\right)$, Dehn-Nielsen-Baer theorem [4] says that $\operatorname{Mod}^{ \pm}\left(S_{g}\right)$ is isomorphic to $\operatorname{Out}\left(\pi_{1}\left(S_{g}\right)\right)$, the outer automorphism group of $\pi_{1}\left(S_{g}\right)$. Ivanov proved that $\operatorname{Mod}^{ \pm}\left(S_{g}\right)$ is the automorphism group of the curve complex [6]. Brock and Margalit showed that $\operatorname{Mod}^{ \pm}\left(S_{g}\right)$ is the automorphism group of the pants complex and it is also the isometry group of the Teichmüller space under the Weil-Petersson metric ([2],[13]). Korkmaz showed that $\operatorname{Mod}^{ \pm}\left(S_{g}\right)$ can be generated by 2 elements, one of which is a Dehn twist [9]. Stukow in [16] proved $\operatorname{Mod}^{ \pm}\left(S_{g}\right)$ is generated by 3 elements of order 2 .

It is an open problem if $\operatorname{Mod}^{ \pm}\left(S_{g}\right)$ can be generated by two torsion elements (See [10], Problem 5.3). In this paper, under the condition that the genus of the surface is at least 5 , we answer this question affirmatively:

Theorem 1.1. For $g \geq 5$, the extended mapping class group $\operatorname{Mod}^{ \pm}\left(S_{g}\right)$ can be generated by 2 torsion elements. One of these generators is of order 2 and the other one is of order $4 g+2$.

\section{Preliminaries}

\section{Notations:}

(a) We use the convention of functional notation, namely, elements of the mapping class group are applied right to left, i.e. the composition $F G$ means that $G$ is applied first.

(b) A Dehn twist means a right-hand Dehn twist.

(c) We denote the curves by lower case letters $a, b, c, d$ (possibly with subscripts) and the Dehn twists about them by the corresponding capital letters $A, B, C, D$. Notationally we do not distinguish a diffeomorphism/curve and its isotopy class.

We recall the following results (see, for instance, section 3.3, 5.1, 7.5 of [4]):

Lemma 2.1. For any $\varphi \in \operatorname{Mod}\left(S_{g}\right)$ and any isotopy classes $a, b$ of simple closed curves in $S_{g}$ satisfying $\varphi(a)=b$, we have:

$$
B=\varphi A \varphi^{-1} \text {. }
$$

Lemma 2.2. For any $\varphi \in \operatorname{Mod}^{ \pm}\left(S_{g}\right) \backslash \operatorname{Mod}\left(S_{g}\right)$ and any isotopy classes $a, b$ of simple closed curves in $S_{g}$ satisfying $\varphi(a)=b$, we have:

$$
B^{-1}=\varphi A \varphi^{-1} \text {. }
$$

Lemma 2.3. Let $a, b$ be two simple closed curves on $S_{g}$. If $a$ is disjoint from $b$, then

$$
A B=B A
$$




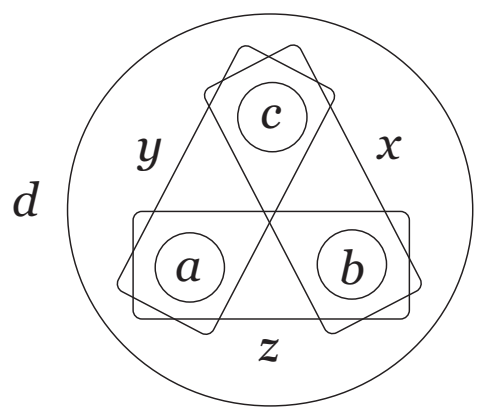

Figure 1

Lemma 2.4 (Lantern relation). Let $a, b, c, d, x, y, z$ be the curves showed in Figure 1 on a genus zero surface with four boundaries. Then

$$
A B C D=X Y Z \text {. }
$$

In other words, since $a, b, c$ are disjoint from $x, y, z$, we have

$$
D=\left(X A^{-1}\right)\left(Y B^{-1}\right)\left(Z C^{-1}\right) .
$$

The elements having the form of $U V^{-1}$ for some disjoint simple closed curves $u, v$ play a crucial role in our proof. The proof of the main result relies on Humphries' theorem:

Theorem 2.5 (Humphries). Let $a_{1}, a_{2}, \ldots a_{2 g}, b$ be the curves as on the lefthand side of Figure 2. Then the mapping class group $\operatorname{Mod}\left(S_{g}\right)$ is generated by $A_{i}$ 's and $B$.

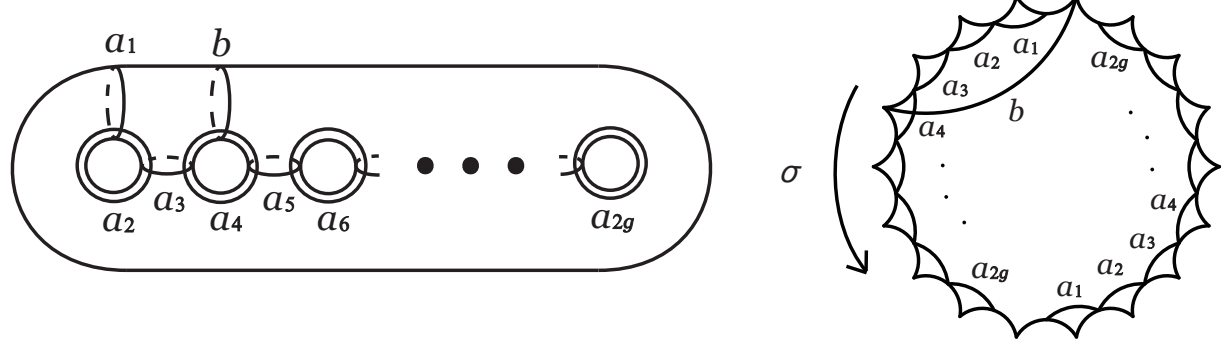

Figure 2

Consider the genus $g$ surface as a regular $(4 g+2)$-gon whose corresponding opposite sides are glued together, as indicated on the right-hand side of Figure 2. We redraw the curves of Humphries' generating set on the righthand side of Figure 2 as follow. The set of curves $a_{i}$ 's on the left-hand side of Figure 2 is a chain of simple closed curves and fills the surface. We find a chain of simple closed curves which also fills the surface on the right-hand side of Figure 2, identify them with $a_{i}$ 's. To see the corresponding curve of $b$, notice that $b$ intersects $a_{4}$ once and is disjoint from other $a_{i}$ 's. Then find such a curve on the right-hand side of Figure 2.

Look at the $(4 g+2)$-gon. There is a natural $2 \pi /(4 g+2)$ rotation preserving the gluing way of the $(4 g+2)$-gon. This rotation induces a period map $\sigma$ 
of the genus $g$ surface. Moreover, $\sigma\left(a_{i}\right)=a_{i+1}$ for $1 \leq i \leq 2 g-1$. Take $a_{0}=\sigma\left(a_{2 g}\right)$. Then we have $a_{1}=\sigma\left(a_{0}\right)$. Under modulo $2 g+1$, we have $a_{k}=\sigma^{k}\left(a_{0}\right)$ for all integer $k$ and $a_{i+1}=\sigma\left(a_{i}\right)$ still holds for all $i$ 's. Similarly, since $\sigma$ is of order $4 g+2$, under modulo $4 g+2$, we take $b_{0}=b$ and $b_{k}=\sigma^{k}(b)$. Then we have $\sigma\left(b_{j}\right)=b_{j+1}$ for all $j$ 's.

To see back the image of $b_{j}$ 's on the left-hand side of Figure 2, since the set of $a_{k}$ 's form a chain of simple closed curves filling the surface, we only need to calculate the geometric intersection number $i\left(b_{j}, a_{k}\right)$ for each $k$ on the right-hand side of Figure 2, then find a curve with the same geometric intersection numbers with $a_{k}$ 's as on the left-hand side of Figure 2. Figure 3 shows this way to think of the images of $b_{j}$ 's. Figure 2 and Figure 3 will be used in the proof of Theorem 3.1 to verify the disjointness between some $a_{i}$ and $b_{j}$.
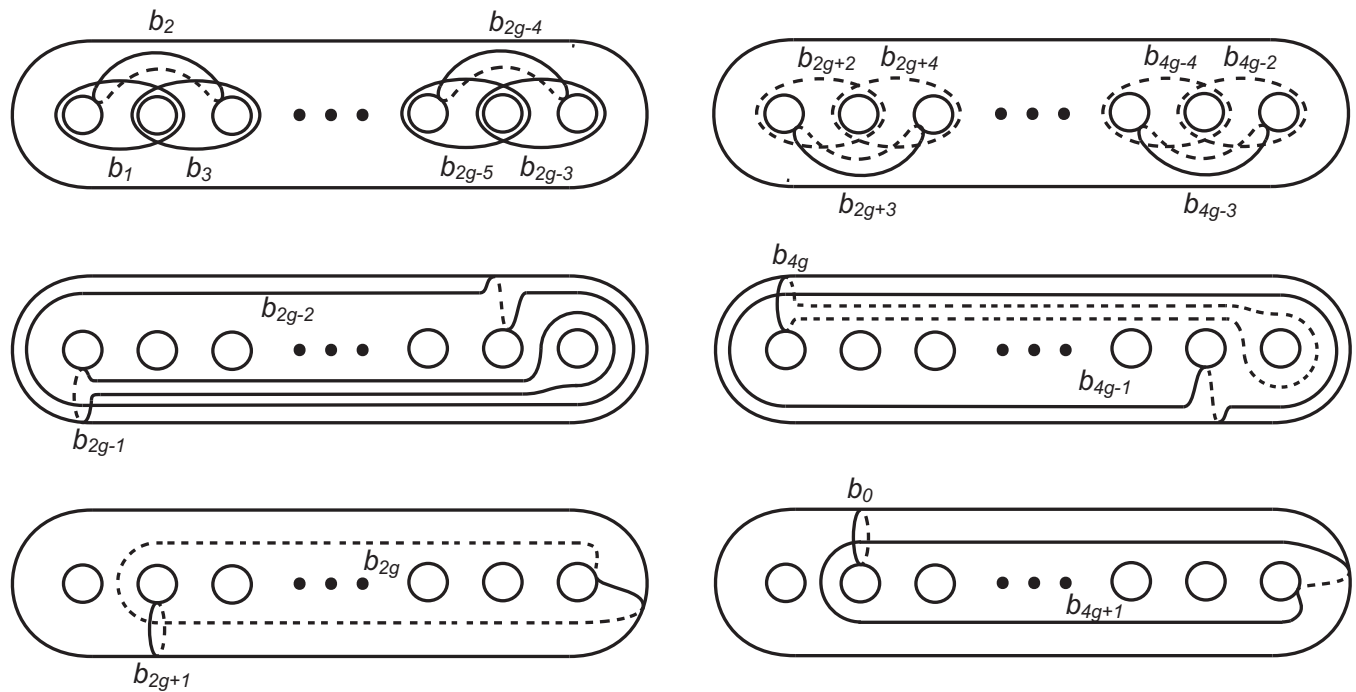

Figure 3

\section{The main Result And the PRoof}

Let $\tau$ be the symmetry of the $(4 g+2)$-gon as on the left-hand side of Figure 4. Then $\tau$ induces an orientation-reversing homeomorphism of the genus $g$ surface. The fixed point set of $\tau$ forms a non-separating curve. A part of such a non-separating curve is along the axis of the symmetry. We still denote the homotopy class of such a homeomorphism as $\tau$. Since $\tau$ preserves the curve $b$, it is easy to check that $(\tau \circ B)^{2}=I d$. 

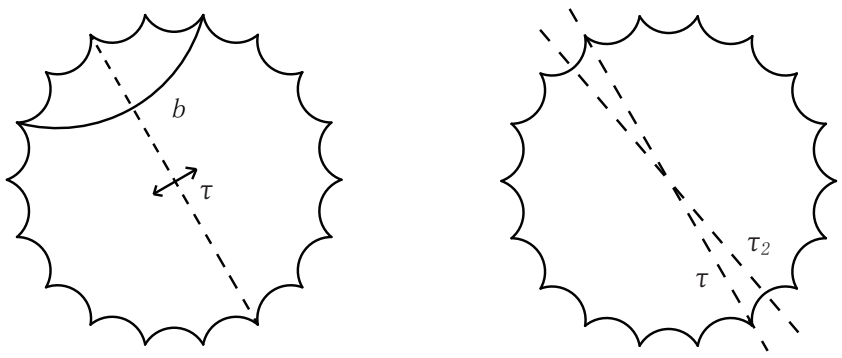

Figure 4

Theorem 3.1. Let $\sigma$ be the element of order $4 g+2$ and $\tau \circ B$ be the element of order 2 as we described above. Then for $g \geq 5$ we have $\operatorname{Mod}^{ \pm}\left(S_{g}\right)=$ $\langle\sigma, \tau \circ B\rangle$.

Remark 3.2. This generating set can also apply to $\operatorname{Mod}^{ \pm}\left(S_{g, 1}\right)$, i.e., the extended mapping class group of the surface with one marked point. This is because the element $\sigma$ and $\tau \circ B$ fix the center point of the $4 g+2$-gon.

Remark 3.3. From Theorem [3.1 we can also deduce that $\operatorname{Mod}^{ \pm}\left(S_{g}\right)$ can be generated by 3 symmetries when $g \geq 5$. The reason is that $\sigma$ is like the rotation of the $(4 g+2)$-gon. In the dihedral group of the $(4 g+2)$-gon, the rotation is the product of two reflections (see the right-hand side of Figure 4). Such reflections of $(4 g+2)$-gon induce orientation reversing order 2 maps $\tau, \tau_{2}$ on the surface. Hence $\operatorname{Mod}^{ \pm}\left(S_{g}\right)=\left\langle\tau \circ B, \tau, \tau_{2}\right\rangle$. So we can get another proof of the result in [16] under the condition $g \geq 5$. Our generating set is different from the generating set in [16]. The fixed-point sets of two of the symmetries in [16] are separating curves. The fixed-point sets of our symmetries are non-separating curves.

Proof of Theorem 3.1. Denote the subgroup generated by $\sigma$ and $\tau \circ B$ as $G$. We prove that $G=\operatorname{Mod}^{ \pm}\left(S_{g}\right)$ in four steps:

Step 1. Under modulo $4 g+2$, the following two conditions are equivalent: (1) integers $i, k$ satisfy $k \in\{4,5,6, \ldots, 4 g-2\} \backslash\{2 g-2,2 g, 2 g+2,2 g+4\}$;

(2) $b_{i}$ is disjoint from $b_{i+k}$. Under such conditions we have $B_{i} B_{i+k}^{-1}$ and $B_{i}^{-1} B_{i+k}$ are in $G$.

Step 2. Under modulo $2 g+1$ for $m$ and modulo $4 g+2$ for $n$, the following two conditions are equivalent: (1) integers $m, n$ satisfy $m \notin\{n, n+4\}$; (2) $a_{m}$ is disjoint from $b_{n}$. Under such conditions we have $A_{m} B_{n}^{-1}$ and $A_{m}^{-1} B_{n}$ are in $G$.

Step 3. Using the lantern relation, prove that for all $k, A_{k} \in G$.

Step 4. Finally, $G=\operatorname{Mod}^{ \pm}\left(S_{g}\right)$.

The proof of Step 1:

It is obvious that $b_{0}$ is disjoint from $b_{k}$ if and only if $k \in\{4,5,6, \ldots, 4 g-$ $2\} \backslash\{2 g-2,2 g, 2 g+2,2 g+4\}$ (see Figure 2 and Figure 3 ). So do $b_{i}$ and 
$b_{i+k}$. Under such conditions, we first prove that $B_{0} B_{k}^{-1} \in G$. Consider the element $\sigma^{k}(\tau \circ B) \sigma^{k}(\tau \circ B)$. In the dihedral subgroup of $\operatorname{Mod}^{ \pm}\left(S_{g}\right)$, $\sigma^{k} \tau \sigma^{k} \tau$ is the identity. After adding the Dehn twist $B$, since $b_{k}$ is disjoint from $b_{0}$, we can easily check that $\sigma^{k}(\tau \circ B) \sigma^{k}(\tau \circ B)=B_{0} B_{k}^{-1}$. For every integer $i$, conjugate $B_{0} B_{k}^{-1}$ by $\sigma^{i}$. Then we have $B_{i} B_{i+k}^{-1}$ is in $G$. The commutativity of $B_{i}$ and $B_{i+k}$ promise $B_{i}^{-1} B_{i+k}$ is in $G$.

The proof of Step 2:

It is obvious that the disjointness between $a_{i}$ and $b_{0}$ is equivalent to $i \notin$ $\{0,4\}$. So $a_{m}$ is disjoint from $b_{n}$ is equivalent to $m \notin\{n, n+4\}$. From these conditions, we also see that $a_{i}$ is disjoint from $b_{0}$ if and only if $a_{4-i}$ is disjoint from $b_{0}$.

By the conjugacy relation, for some $i, k, m, n$ satisfying the conditions in Step 1 and Step 2, if there exists $\varphi \in G$ and a pair of disjoint curves $\left(b_{i}, b_{i+k}\right)$ such that $\varphi:\left(b_{i}, b_{i+k}\right) \mapsto\left(a_{m}, b_{n}\right)$, since $B_{i} B_{i+k}^{-1}$ is in $G, A_{m} B_{n}^{-1}$ is also in $G$. We need to find such a $\varphi \in G$.

The proofs in odd genus case and in even genus case are slightly different. Suppose first that the genus $g$ is odd. See Figure 5.

(1)

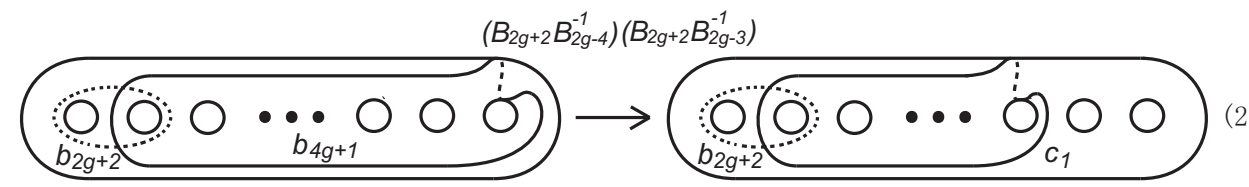

(3)

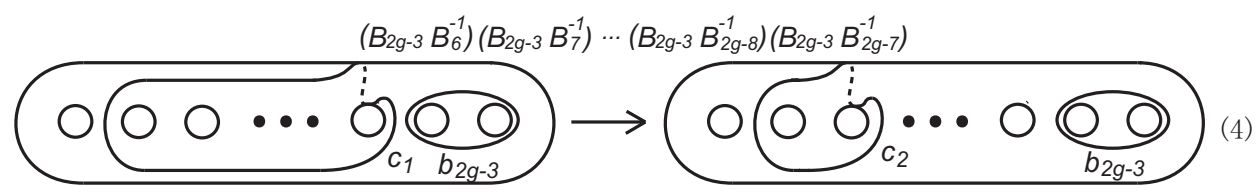

(6)

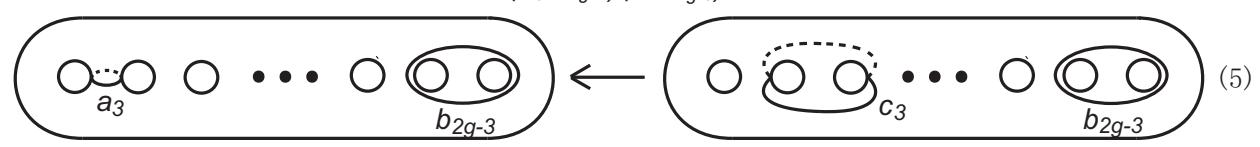

Figure 5

By Step $1, B_{2 g+2} B_{4 g+1}^{-1}$ is in $G$. If $g \geq 5, b_{2 g+2}$ is disjoint from $b_{2 g-3}, b_{2 g-4}$. Both $B_{2 g+2} B_{2 g-4}^{-1}$ and $B_{2 g+2} B_{2 g-3}^{-1}$ are in $G$. The element $\left(B_{2 g+2} B_{2 g-4}^{-1}\right)$. $\left(B_{2 g+2} B_{2 g-3}^{-1}\right)$ maps the pair of curves $\left(b_{2 g+2}, b_{4 g+1}\right)$ to the pair of curves $\left(b_{2 g+2}, c_{1}\right)$ as in Figure $5(2)$. Hence $B_{2 g+2} C_{1}^{-1}$ is in $G$. Now $B_{2 g-3} B_{2 g+2}^{-1}$ is also in $G$. So $B_{2 g-3} C_{1}^{-1}$ is in $G$.

The curve $b_{2 g-3}$ is disjoint from $b_{2 g-7}, b_{2 g-8}, \ldots, b_{7}, b_{6}$. The elements $\left(B_{2 g-3} B_{6}^{-1}\right),\left(B_{2 g-3} B_{7}^{-1}\right) \ldots\left(B_{2 g-3} B_{2 g-8}^{-1}\right),\left(B_{2 g-3} B_{2 g-7}^{-1}\right)$ are in $G$. Then 
THE EXTENDED MAPPING CLASS GROUP CAN BE GENERATED BY TWO TORSION\$

their product $\left(B_{2 g-3} B_{6}^{-1}\right) \cdot\left(B_{2 g-3} B_{7}^{-1}\right) \ldots\left(B_{2 g-3} B_{2 g-8}^{-1}\right) \cdot\left(B_{2 g-3} B_{2 g-7}^{-1}\right)$ maps the pair of curves $\left(b_{2 g-3}, c_{1}\right)$ to the pair of curves $\left(b_{2 g-3}, c_{2}\right)$ as in Figure 5 (4). We have $B_{2 g-3} C_{2}^{-1}$ is in $G$.

The curve $b_{2 g-3}$ is disjoint from $b_{3}$ and $b_{2}$. The elements $\left(B_{2 g-3} B_{2}^{-1}\right)$, $\left(B_{2 g-3} B_{3}^{-1}\right)$ are in $G$. Their product $\left(B_{3} B_{2 g-3}^{-1}\right) \cdot\left(B_{2} B_{2 g-3}^{-1}\right)$ maps the pair of curves $\left(b_{2 g-3}, c_{2}\right)$ to the pair of curves $\left(b_{2 g-3}, a_{3}\right)$. So we get that $B_{2 g-3} A_{3}^{-1}$ is in $G$. Its inverse $A_{3} B_{2 g-3}^{-1}$ is also in $G$.

Suppose now that $g$ is even. See Figure 6 .

(1)

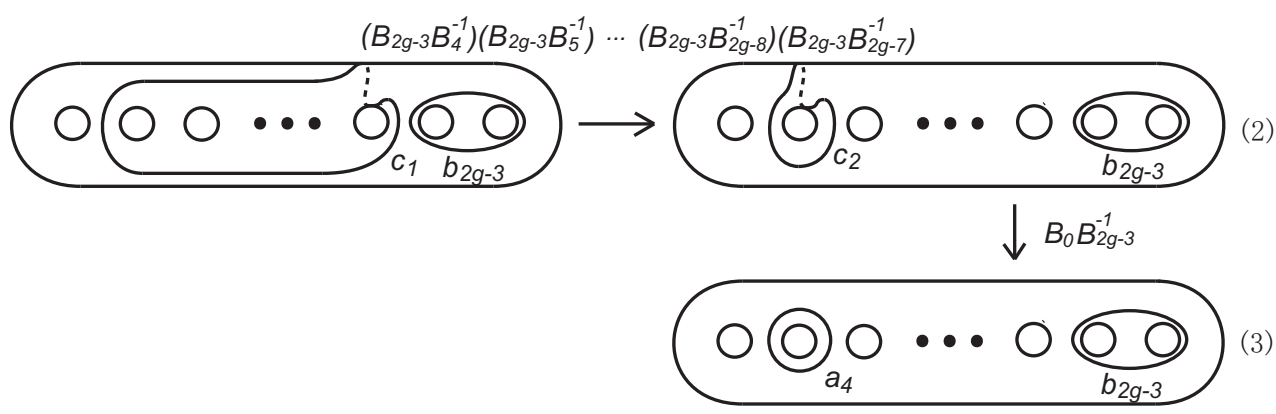

Figure 6

In this case, the proof of $B_{2 g-3} C_{1}^{-1} \in G$ is the same as the odd genus case. The element $\left(B_{2 g-3} B_{4}^{-1}\right) \cdot\left(B_{2 g-3} B_{5}^{-1}\right) \ldots\left(B_{2 g-3} B_{2 g-8}^{-1}\right) \cdot\left(B_{2 g-3} B_{2 g-7}^{-1}\right)$ maps the pair of curves $\left(b_{2 g-3}, c_{1}\right)$ to the pair of curves $\left(b_{2 g-3}, c_{2}\right)$ as in Figure $6(2)$. The element $B_{0} B_{2 g-3}^{-1}$ maps the pair of curves $\left(b_{2 g-3}, c_{2}\right)$ to the pair of curves $\left(b_{2 g-3}, a_{4}\right)$. So we get that $B_{2 g-3} A_{4}^{-1}$ is in $G$. Its inverse $A_{4} B_{2 g-3}^{-1}$ is also in $G$.

The element $\sigma^{2 g+5}$ maps $\left(a_{3}, b_{2 g-3}\right)$ to $\left(a_{2 g+8}, b_{0}\right)$ when $g$ is odd and maps $\left(a_{4}, b_{2 g-3}\right)$ to $\left(a_{2 g+9}, b_{0}\right)$ when $g$ is even. We have $A_{i} B_{0}^{-1}=B_{0}^{-1} A_{i} \in G$ for some $i$ where $a_{i}$ is disjoint from $b_{0}$.

Notice that $\tau \sigma^{k} \tau$ maps $a_{i}$ to $a_{i-k}$, hence $A_{i-k}=\left(\tau \sigma^{k} \tau\right) A_{i}\left(\tau \sigma^{k} \tau\right)^{-1}$. So as long as $a_{i-k}$ is disjoint from $b_{0}$, we have

$$
\begin{aligned}
& \left(\tau \circ B_{0}\right) \sigma^{k}\left(\tau \circ B_{0}\right)\left(A_{i} B_{0}^{-1}\right) \sigma^{k}=\left(B_{0}^{-1} \circ \tau\right) \sigma^{k}\left(\tau \circ A_{i}\right) \sigma^{k} \\
= & B_{0}^{-1}\left(\tau \sigma^{k} \tau\right) A_{i} \sigma^{k}=B_{0}^{-1}\left(A_{i-k} \tau \sigma^{k} \tau\right) \sigma^{k}=B_{0}^{-1} A_{i-k}=A_{i-k} B_{0}^{-1} .
\end{aligned}
$$

Taking all possible $k$ and conjugating by $\sigma^{n}$, we have all $A_{m} B_{n}^{-1} \in G$ for $m \notin\{n, n+4\}$. The commutativity of $A_{m}$ and $B_{n}$ promises $A_{m}^{-1} B_{n}$ is in $G$.

The proof of Step 3:

See Figure 7. There is a natural lantern lying on the surface, bounded by $a_{1}, a_{3}, a_{5}$ and $f$. By the lantern relation, we have $B_{0} B_{2} E=A_{1} A_{3} A_{5} F$, or $A_{1}=\left(B_{0} A_{3}^{-1}\right)\left(B_{2} A_{5}^{-1}\right)\left(E F^{-1}\right)$, where $e$ and $f$ are the curves showed in Figure 7. The elements $B_{0} A_{3}^{-1}$ and $B_{2} A_{5}^{-1}$ are the inverses of $A_{3} B_{0}^{-1}$ and 
$A_{5} B_{2}^{-1}$ respectively. By the result of step 2 , they are in $G$. We only need to prove $E F^{-1}$ is also in $G$.
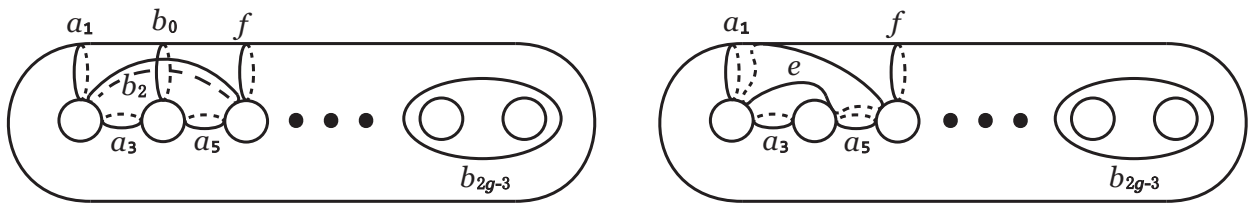

Figure 7

When $g \geq 5, b_{2 g-3}$ is disjoint from $b_{0}, \ldots, b_{3}, a_{1}, \ldots, a_{6}, e, f$. Notice $E F^{-1}=\left(E B_{2 g-3}^{-1}\right)\left(B_{2 g-3} F^{-1}\right)$. We verify $E B_{2 g-3}^{-1}$ and $B_{2 g-3} F^{-1}$ are in $G$. By the previous steps, The element $\left(B_{2 g-3} B_{3}^{-1}\right) \cdot\left(A_{6} B_{2 g-3}^{-1}\right) \cdot\left(A_{5} B_{2 g-3}^{-1}\right)$ - $\left(A_{4} B_{2 g-3}^{-1}\right)$ is in $G$ and maps the pair of curves $\left(b_{2 g-3}, b_{0}\right)$ to $\left(b_{2 g-3}, f\right)$. Notice $B_{2 g-3} B_{0}^{-1}$ is in $G$. So $B_{2 g-3} F^{-1}$ is in $G$. The element $\left(A_{2} B_{2 g-3}^{-1}\right)$ - $\left(A_{1} B_{2 g-3}^{-1}\right) \cdot\left(A_{4}^{-1} B_{2 g-3}\right) \cdot\left(B_{1} B_{2 g-3}^{-1}\right)$ maps the pair of curves $\left(b_{2 g-3}, a_{5}\right)$ to $\left(b_{2 g-3}, e\right)$. Notice $B_{2 g-3} A_{5}^{-1}$ is in $G$. So $B_{2 g-3} E^{-1}$ is in $G$. We now get $E F^{-1}=\left(E B_{2 g-3}^{-1}\right)\left(B_{2 g-3} F^{-1}\right)$ is in $G$.

The proof of Step 4:

The fact that the elements $A_{1}$ and $A_{1} B_{0}^{-1}$ are in $G$ means that $B_{0} \in G$. Now all the curves $a_{i}$ 's are in the same orbit of $\sigma$. So do $b_{j}$ 's. So all the $A_{i}$ 's and $B_{j}$ 's are in $G$. These include Humphries' generators of $\operatorname{Mod}\left(S_{g}\right)$. So $G$ contains $\operatorname{Mod}\left(S_{g}\right)$. Finally, one of the generator $\tau \circ B$ is an orientationreversing mapping class. Hence $G=\operatorname{Mod}^{ \pm}\left(S_{g}\right)$.

Remark 3.4. The idea of Step 2 and Step 3 in the above proof is based on the method in [9] to prove $\operatorname{Mod}\left(S_{g}\right)$ is generated by two torsions of order $4 g+2$.

\section{REFERENCES}

[1] T. E. Brendle and B. Farb. Every mapping class group is generated by 3 torsion elements and by 6 involutions. J. Algebra 278 (2004), 187-198.

[2] J. Brock and D. Margalit. Weil-Petersson isometries via the pants complex. Proc. Amer. Math. Soc., 135(3):795-803 (electronic), 2007.

[3] M. Dehn. Papers on group theory and topology (Springer-Verlag, New York, 1987) (Die Gruppe der Abbildungsklassen, Acta Math. Vol. 69 (1938), 135206).

[4] B. Farb and D. Marglait. A Primer on Mapping Class Groups. Princeton Math. Ser., (Princeton University Press, 2012), 623-658.

[5] S. P. Humphries. Generators for the mapping class group. Topology of lowdimensional manifolds. Proc. Second Sussex Conf. Chelwood Gate 1977 Lecture Notes in Math. 722 (Springer, 1979), 44-47.

[6] N. V. Ivanov. Automorphism of complexes of curves and of Teichmuller spaces. Internat. Math. Res. Notices, (14):651-666, 1997. 
THE EXTENDED MAPPING CLASS GROUP CAN BE GENERATED BY TWO TORSIONg

[7] D. Johnson. The structure of Torelli group I: A finite set of generators for I. Ann. of Math. 118 (1983), 423-442.

[8] M. Kassabov. Generating Mapping Class Groups by Involutions. arXiv:math.GT/0311455 v1 25 Nov 2003.

[9] M. Korkmaz. Generating the surface mapping class group by two elements. Trans. Amer. Math. Soc. 357 (2005), 3299-3310.

[10] M. Korkmaz. Minimal generating sets for the mapping class group of a surface. Handbook of Teichmüller theory. Volume III, 441-463, IRMA Lect. Math. Theor. Phys., 17, Eur. Math. Soc., Zürich, 2012.

[11] W. B. R. Lickorish. A finite set of generators for the homeotopy group of a 2-manifold. Proc. Camb. Phils. Soc. 60 (1964), 769-778.

[12] F. Luo. Torsion Elements in the Mapping Class Group of a Surface. arXiv:math.GT/0004048 v1 8 Apr 2000.

[13] D. Margalit. Automorphisms of the pants complex. Duke Math. J., 121(3):457-479, 2004.

[14] N. Monden. Generating the mapping class group by torsion elements of small order. Mathematical Proceedings of the Cambridge Philosophical Society, 154 (2013), pp 41-62, doi:10.1017/S0305004112000357.

[15] J. D. McCarthy and A. Papadopoulos. Involutions in surface mapping class groups.Enseign. Math.(2) 33 (1987), no. 3-4, 275-290. 456.

[16] M. Stukow. The extended mapping class group is generated by 3 symmetries. C. R. Math. Acad. Sci. Paris 338 (2004), no. 5, 403-406.

[17] B. Wajnryb. Mapping class group of a surface is generated by two elements. Topology, 35(2), 377-383, 1996.

South China University of Technology, Guangzhou 510640, P.R.China

E-mail address: scxmdu@scut.edu.cn 